\title{
Perfusion Computed Tomography in Predicting Treatment Response of Advanced Esophageal Squamous Cell Carcinomas
}

\author{
Ming-Huan $\mathrm{Li}^{1 *}$, Dong-Ping Shang ${ }^{2}$, Chen Chen ${ }^{1}$, Liang $\mathrm{Xu}^{2}$, Yong Huang ${ }^{2}, \mathrm{Li}$ \\ Kong ${ }^{1}$, Jin-Ming Yu ${ }^{1 *}$
}

\begin{abstract}
Background: The purpose of this study was to prospectively evaluate the predictive value of perfusion computed tomography (CT) for response of local advanced esophageal carcinoma to radiotherapy and chemotherapy. Materials and Methods: Before any treatment, forty-three local advanced esophageal squamous cell carcinomas were prospectively evaluated by perfusion scan with 16-row CT from June 2009 to January 2012. Perfusion parameters, including perfusion (BF), peak enhanced density (PED), blood volume (BV), and time to peak (TTP) were measured using Philips perfusion software. Seventeen cases received definitive radiotherapy and 26 received concurrent chemo-radiotherapy. The response was evaluated by CT scan and esophagography. Differences in perfusion parameters between responders and non-responders were analyzed, and ROCs were used to assess predictive value of the baseline parameters for treatment response. Results: There were 25 responders (R) and 18 non-responders (NR). Responders showed significantly higher BF (R:34.1 ml/100g/min vs NR: 25.0 $\mathrm{ml} / 100 \mathrm{~g} / \mathrm{min}, p=0.001), \mathrm{BV}(23.2 \mathrm{ml} / 100 \mathrm{~g} v s 18.3 \mathrm{ml} / 100 \mathrm{~g}, p=0.009)$ and PED $(32.5 \mathrm{HU}$ vs $28.32 \mathrm{HU}, P=0.003)$ than non-responders. But the baseline TTP (R: 38.2s vs NR: 44.10s, $p=0.172)$ had no difference in the two groups. For baseline BF, a threshold of $36.1 \mathrm{ml} / 100 \mathrm{~g} / \mathrm{min}$ achieved a sensitivity of $56 \%$, and a specificity of $94.4 \%$ for detection of clinical responders from non-responders. Conclusions: The results suggest that the perfusion CT can provide some helpful information for identifying tumors that may respond to radio-chemotherapy.
\end{abstract}

Keywords: Esophageal squamous cell carcinoma - perfusion CT - radiotherapy - chemotherapy - response

Asian Pac J Cancer Prev, 16 (2), 797-802

\section{Introduction}

Esophageal cancer is one of the most deadly cancers with a rapidly rising incidence. More than $50 \%$ of esophageal cancers are diagnosed at locally advanced stages, which are generally treated using multimodal therapies. Chemo-radiotherapy (CRT) followed by esophagectomy has become a widespread treatment protocol since several favorable studies were reported (Bedenne et al., 2007; Kelsen et al., 2007; van Hagen et al., 2012; Wang et al., 2012). Studies have demonstrated that the survival benefit of pre-operative therapies is restricted within the responders. But there remains the fact that only a subset of patients obtain a major or complete response after radiation and chemotherapy (Stahl et al., 2005; Piessen et al., 2007; Kersting et al., 2009). It is reported that FDG PET can provide reproducible data for early response assessment in esophageal cancer by depicting a reduction in the metabolic activity of the tumor (Zhu et al., 2012; Miyata et al., 2014). Till now, for esophageal cancer, we do not have good markers to predict response to therapy and avoid any time delay in non-responders.
Tumor angiogenesis is essential for cancer growth and provides an attractive target for oncologic diagnoses and therapies. Perfusion computed tomography (CT) is an useful imaging tool that provides both qualitative and quantitative information regarding tumor angiogenesis (Laking et al., 2006). Oxygen and drug delivery to tumor tissues appears to rely on a network of microvessels. The rationale of the potential role of perfusion CT in predicting response to nonsurgical therapies is that it is substantially influenced by tissue perfusion and local oxygen delivery (Moulder et al., 1987; Tsai et al., 2003).

It has been shown that squamous cell carcinomas of esophagus have increased values of perfusion $(\mathrm{BF})$, peak enhanced density (PED), blood volume (BV), and shorter time to peak (TTP) compared with normal esophagus (Yin et al., 2008; Li et al., 2009). The changes of perfusion CT parameters are probably due to the tumor neovascularity as demonstrated by angiography and histochemical analysis of tumor specimens (Goh et al., 2006; Chen et al., 2011). The present study aimed at estimating the value of perfusion $\mathrm{CT}$ on predicting the response after radio (chemo)-therapy in patients with local advanced esophageal cancer. 


\section{Materials and Methods}

\section{Patients}

The study was approved by the ethics committee of Shandong Cancer Hospital and Institute; written informed consent was obtained from all participants. Patients eligible for this study had to have histologically proven carcinoma of esophagus. No prior treatment was allowed. Eligibility also required the involved esophageal wall thickness more than $10 \mathrm{~mm}$ on CT scans. All subjects should be $\leq 75$ years of age with an Eastern Cooperative Oncology Group performance status 0 to 2. Patients were excluded if they had distant metastasis and if they had a known allergy to intravenous contrast material. Local tumor staging was based on findings at endoesophageal ultrasonography and CT.

\section{Perfusion CT protocol}

All patients underwent baseline perfusion CT on 1-7 days before the beginning of radiotherapy. Scanning was performed with a 16-slice Philip Brilliance big bore CT scanner (Philips Medical Systems; the Netherlands).) that had $16 \times 1.5 \mathrm{~mm}$ detectors. The CT imaging protocol involved three steps: a scout scan, a study of the thoracic and abdomen/neck without contrast enhancement, and a dynamic study with contrast enhancement used for perfusion CT. The non-enhanced study was performed initially to identify the location of the tumor for planning purposes. The following parameters were used: slice thickness, $3 \mathrm{~mm}$; speed, 30mm/sec; pitch, 0.94; $120 \mathrm{kV}$; $200 \mathrm{~mA}$; scan field of view, $300 \mathrm{~mm}$; and matrix, $512 \times 512$ $\mathrm{mm}$. The target sections (a $24 \mathrm{~mm}$ scanning range) were selected by two authors ( $\mathrm{Li}$ and Shang), and the range was chosen to include the maximum tumor area visible. For the dynamic study, CT was performed with no scanning delay after injection of a $0.8 \mathrm{~mL} / \mathrm{kg}$ bolus of contrast material administered at a rate of $6-7 \mathrm{~mL} / \mathrm{sec}$ via an 18 -gauge cannula in the ante cubital vein. The cycle time was $2 \mathrm{~s}$ (scan time $0.5 \mathrm{~s}+$ interval $1.5 \mathrm{~s}$ ), $120 \mathrm{kV} ; 200 \mathrm{~mA}$; scan field of view, $300 \mathrm{~mm}$; matrix, $512 \times 512 \mathrm{~mm}$ ). Dynamic scanning was maintained during the bolus injection of contrast material and continued for 80 seconds.

\section{Image analysis}

The images and data obtained were transferred to an image processing workstation (Extended BrillianceTM Workspace V3.5 .0, Philips Healthcare) and commercially available software (Brilliance perfusion 2.1.1, Perfusion CT; Philips Healthcare) was used to calculate perfusion parameters. The analytical method used in this software was based on the maximum slope method. For the derivation of functional maps of these perfusion parameters, the arterial input curve of the contrast medium concentration is required and we obtained this arterial input curve from a ROI in the aorta (Figure 1.A, B). If the tumor was so high that we could not obtain a ROI of the aorta, we obtained the arterial input curve from a ROI in the carotid artery. Time attenuation curves (TAC) and kinetic parameters for the input artery and the tumor were generated automatically by the commercial software. The major kinetic parameters were perfusion $(\mathrm{BF}$, in $\mathrm{ml}$ per
$100 \mathrm{~g}$ per min, Figure 1.C), peak enhancement density (PED, in Hounsfield units, Figure1.D), blood volume (BV, in $\mathrm{ml}$ per $100 \mathrm{~g}$, Figure1.E), and time to peak (TTP, in seconds, Figure 1.F). A single axial slice that best visualized the tumor was chosen from the 8 axial slices available by two authors (Li and Shang).

\section{Treatment}

Chemotherapy began on day 1 , concurrent with the beginning of radiation. The chemotherapeutic regimen consisted of two cycles of cisplatin $\left(75 \mathrm{mg} / \mathrm{m}^{2} /\right.$ day on day 1 or $25 \mathrm{mg} / \mathrm{m}^{2} /$ day on day 1-3) and fluorouracil (500 $\mathrm{mg} / \mathrm{m} 2 /$ day as a continuous infusion on day 1 through day 5) every 21 days. For radiation, the involved field irradiation was used. The tumor volume was defined as any visible esophageal lesion and clinical involved node on the image. The prescribed dose to plan target volume was $60 \mathrm{~Gy}$ to $64 \mathrm{~Gy}$. All the patients received definitive radiotherapy with (26 cases) or without (17 cases) concurrent chemotherapy.

\section{Evaluation of response and survival}

Evaluation of the primary tumor was performed by CT and esophagography after 2-4 weeks of the completion of RT. The evaluation was based on a comparison of preand post-treatment imaging study findings. Complete response was defined as the disappearance of all signs and symptoms of the tumor. Partial response was defined as a $\geq 50 \%$ decrease in tumor volume. STable disease was defined as a $<50 \%$ decrease or $<25 \%$ increase in tumor volume. Progressive disease was defined as no significant change in tumor mass or a $>25 \%$ increase in tumor volume. Patients who showed a complete or partial response, were categorized as clinical responders. The remaining patients with either sTable or progressive disease were categorized as clinical non-responders.

The date of local or distant failure was defined as the earliest date at which disease progression was confirmed clinically, with imaging, or through biopsy. Overall survival was measured from the date of the baseline perfusion CT scan to the date of death. Patients who were alive on the closeout date had their survival data censored to that date.

\section{Statistical analysis}

The mean and SD of BF, PED, TTP, and BV measurements in the study subjects were determined by independent sample Student's t test (statistical software package: SPSS 11.5). The perfusion parameters before treatment were compared between clinical responders and non-responders by Mann-Whitney U test. The Spearman correlation analysis was used to detect any significant correlation among the perfusion parameters. A receiver operating characteristic curve was used to determine the best cutoff value of perfusion parameters for differentiation between responders and non-responders. The Kaplan-Meier method and log rank test were applied to estimate survival probabilities and compare survival, respectively. The $\mathrm{P}$ value cutoff for statistical significance was set at 0.05 (two-tailed level of significance). 


\section{Results}

Forty-three patients who met the inclusion criteria (36 men and 7 women; median age, 62 years; range, 42-75 years) were enrolled in the study from June 2009 to January 2012. All patients tolerated the perfusion CT examinations well, with no side effects due to high-flow injection of the contrast agent. The patients' characteristics were listed in Table 1.

\section{Baseline perfusion parameters and response.}

Overall responses, including complete and partial response, were observed in 25 cases $(58.1 \%)$, with no response in remaining 18 cases $(41.9 \%)$. There were significant differences in baseline $\mathrm{BF}(p=0.001)$, PED $(p=0.004)$, and BV ( $p=0.009)$ between the responders and non-responders, respectively (Table 2 and Figureure2$\mathrm{A}, \mathrm{B}, \mathrm{C})$. No significantly difference was found between the responders and non-responders for TTP ( $p=0.172$, Figureure2-D). The Spearman correlation analysis of the functional parameters revealed statistically significant correlations between $\mathrm{BF}$ and $\mathrm{BV}(\mathrm{r}=0.553, \mathrm{P}=0.000)$, $\mathrm{BF}$ and PED ( $\mathrm{r}=0.427, p=0.004), \mathrm{BF}$ and TTP $(\mathrm{r}=-0.473$, $\mathrm{P}=0.001)$, and PED and BV $(\mathrm{r}=0.698, p=0.000)$. But the TTP was not significant correlated with either PED $(\mathrm{r}=0.033, p=0.853)$ or BV $(\mathrm{r}=-0.062, \mathrm{P}=0.692)$.

\section{Model for predicting response}

The ROC of the functional parameters showed a significant predictive value of $\mathrm{BF}$ for response (BF=36.10/100g/min, sensitivity/specificity, 56\%/99.4\%; $\mathrm{ROC}=0.813, p=0.001$ (Figure 3A). PE and BV also held predictive values for response $(\mathrm{PE}=26.65 \mathrm{Hu}$, sensitivity/specificity, $96 \% / 67 \%$; ROC $=0.762, \mathrm{P}$ $=0.004 ;$ and $\mathrm{BV}=19.55 \mathrm{ml} / 100 \mathrm{~g}$, sensitivity/specificity, $80 \% / 61 \%$; ROC $=0.734, \mathrm{P}=0.009$; respectively) (Figure $3 \mathrm{~B}$ and $\mathrm{C}$ ). As mentioned above, there were statistically significant correlations among these functional parameters. Therefore, we used BF as the predictor of response to CRT. For baseline BF, when the cut-off point was set at $36.10 \mathrm{ml} / 100 \mathrm{~g} / \mathrm{min}$, sensitivity was $56 \%$, and specificity was $94.4 \%$ for detection of clinical responsers.

\section{Table 1. Patient Characteristics}

\begin{tabular}{lr}
\hline Characteristics & No. of patients \\
\hline Age: $60 \mathrm{y} />60 \mathrm{y}$ & $6 / 37$ \\
Gender: Male /female & $36 / 7$ \\
Location: Upper/Middle/Lower & $13 / 19 / 11$ \\
Length: $<5 \mathrm{~cm} />5 \mathrm{~cm}$ & $19 / 24$ \\
T stage: T3/T4 & $23 / 20$ \\
N status: N0/N+ & $15 / 28$ \\
\hline
\end{tabular}

Table 2. Response and Perfusion Parameters

\begin{tabular}{lccc}
\hline & $\mathrm{R}$ & $\mathrm{NR}$ & $\begin{array}{c}\text { Mannu } \\
\text { white U test } \\
\end{array}$ \\
& $\mathrm{n}=25$ & $\mathrm{n}=18$ & $p$ \\
\hline $\mathrm{BF}(\mathrm{ml} / 100 \mathrm{~g} / \mathrm{min})$ & $34.12 \pm 8.84$ & $24.96 \pm 7.59$ & 0.001 \\
$\mathrm{PED}(\mathrm{Hu})$ & $32.49 \pm 4.37$ & $28.32 \pm 7.25$ & 0.004 \\
$\mathrm{TTP}(\mathrm{s})$ & $38.18 \pm 10.82$ & $44.10 \pm 5.27$ & 0.172 \\
$\mathrm{BV}(\mathrm{ml} / 100 \mathrm{~g})$ & $23.20 \pm 5.27$ & $18.30 \pm 5.69$ & 0.009 \\
\hline
\end{tabular}

\section{Baseline BF and survival}

The follow-up closeout date was March 31, 2013. The median follow-up time was 14 months (range, 4-32 months) The median OS time was 9 months (95\%CI, 7.2--10.7months). The survival period of the responder (10 months, 95\% CI, 8.6--11.4months) group was significantly better than that of the non-responder (7months, 95\% CI, 5.7--8.2months) group ( (log rank chi square, 11.94;
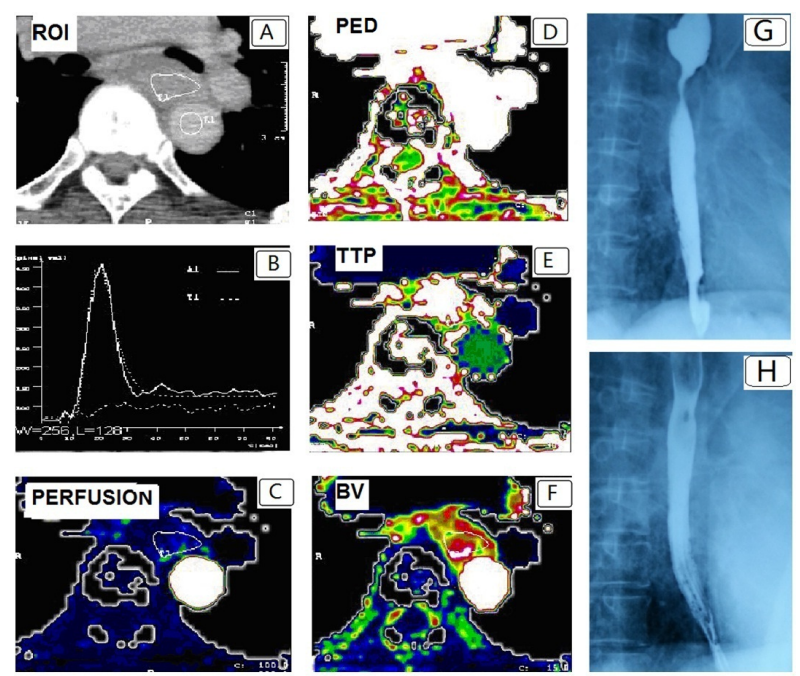

Figure 1. A 62-year-old Man with Squamous cell Carcinoma in the Middle Thoracic Portion of Esophagus. A circular ROI (a) is drawn around descending aorta (A1) to yield the perfusion maps and the arterial time attenuation curves(B) and another one (A) for the tumor (T1) is drawn around the thickened esophageal wall. Among the time attenuation curves, the flatter curve at the bottom of the graph (B) represents the tumor-derived curve. Color parametric maps of Perfusion/BF (C), PED(D), BV(E), and TTP (F) indicate the value of the parameters ranging from high (red) to low (blue). The parameter values correspond to $37.8 \mathrm{ml} / 100 \mathrm{~g} / \mathrm{min}, 39 \mathrm{Hu}$, $26.3 \mathrm{ml} / 100 \mathrm{~g}$, and $34 \mathrm{~s}$, respectively. The clinical response of the tumor to CRT was categorized as responder (G:Pre-CRT; H: Post-CRT)
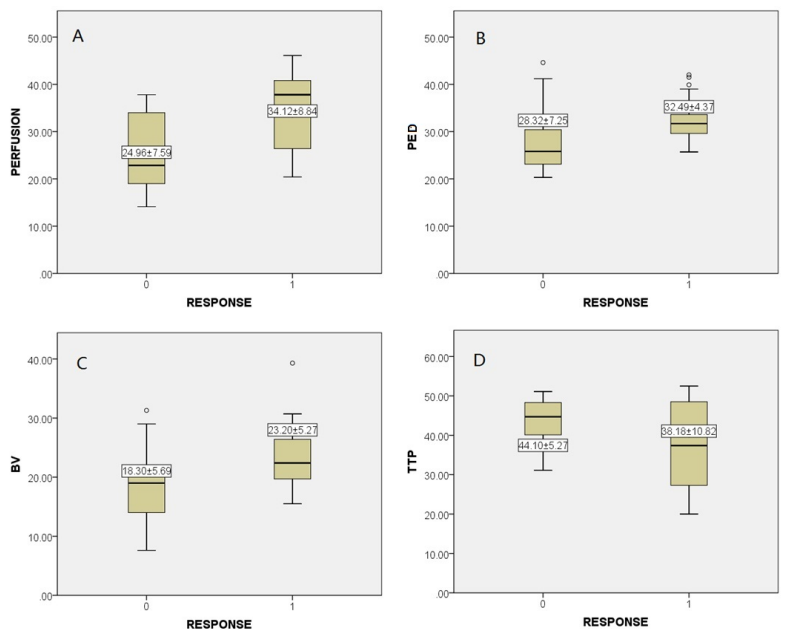

Figure 2. There were Significant Differences in Baseline Perfusion/BF (A, $p=0.001)$, PED $(B, p=0.004)$, and $\mathrm{BV}(\mathrm{C}, p=0.009)$ between Groups of $\operatorname{Responder}(0)$ and Non-responder(1), Respectively. But no significantly difference was found for baseline TTP $(\mathrm{D}, \mathrm{P}=0.172)$ 


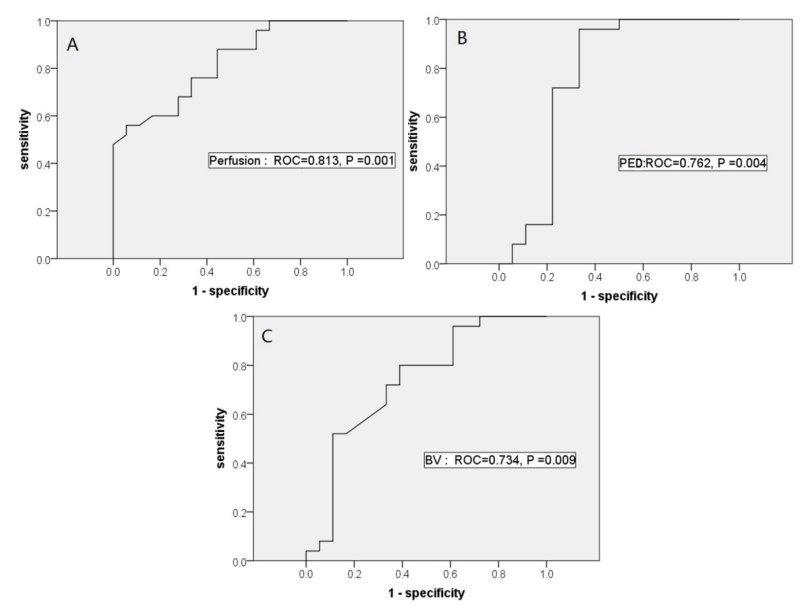

Figure 3. The Area Under the ROC Curves for the Predictive Model. (A: PF; B: PED; C: BV.)
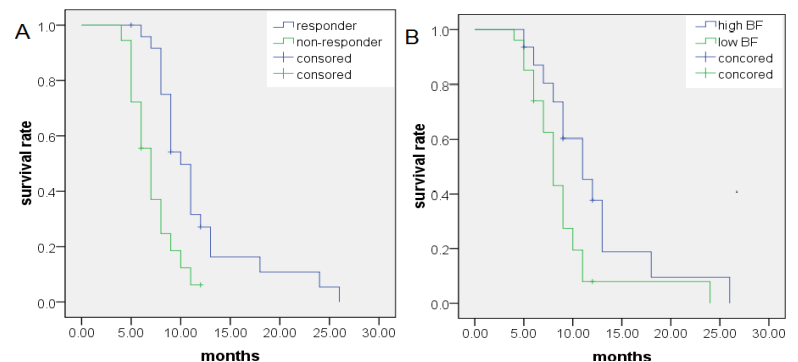

Figure 4. Survival curves were plotted by KaplanMeier methods separately by response (A) and BF (B).The median survival period of the responder group was significantly better than that of the non-responder group (10 months vs 7 months; $\log$ rank chi square, $11.94 ; p=0.001$, Figure 4A). The median survival periods were 8.0 months and 11.0 months for low and high BF groups, respectively (log rank chi square, 5.36; $p=0.021$, Figure 4B)

$p=0.001$, Figure. 4A) The Kaplan-Meier analysis showed that when the patient population was dichotomized according to the $36.10 \mathrm{ml} / 100 \mathrm{~g} / \mathrm{min}$ of tissue $\mathrm{BF}$ value, the patients with perfusion above this cutoff value had a longer OS than the patients with hypo-perfused tumors. The median survival periods were 8.0 months (95\% CI, 7.0--8.9months) and 11.0 months (95\%CI, 7.5--14.4months) for low and high BF groups, respectively (log rank chi square, 5.36; $p=0.021$, Figure. 4B).

\section{Discussion}

In the present study, we examined the usefulness of perfusion $\mathrm{CT}$ on predicting of response in patients with local advanced esophageal squamous cell carcinoma who received radiation and chemotherapy. Our study suggested that perfusion CT can provide some helpful information for the prediction of the response and survival. Radiochemotherapy may be more effective in esophageal squamous cell carcinoma with higher baseline BF, BV and PED. These findings confirmed by those results of other perfusion studies. Hayano et al also found that clinical responders showed significantly higher baseline blood flow (BF), significantly higher baseline blood volume (BV) and a significantly shorter baseline mean transit time (MTT)than non-responders in esophageal squamous cell carcinoma (Hayano et al., 2007). In another esophageal cancer study, it reported that high $\mathrm{BF}$ and low MTT correlated significantly with a good response by neoadjuvant therapies, while BV did not (Makari et al., 2007). In an oropharynx squamous cell carcinoma study, it was found that the baseline $\mathrm{BF}, \mathrm{BV}$, and permeability surface area product (PS) were significantly higher, whereas mean transit time (MTT) was significantly lower in the responders than in the non-responders (Bisdas et al., 2010). Bellomi reported that in rectal carcinoma the baseline $\mathrm{BF}$ and $\mathrm{BV}$ in patients who did not respond to therapy were significantly lower than in responders (Bellomi et al., 2007). Though different perfusionmeasuring algorithms were used, these studies reported potential value of perfusion CT in predicting the response of tumors to chemo-radiotherapy. Especially, baseline $\mathrm{BF}$ (perfusion) is significantly higher in responders than in non-responders to chemotherapy and radiotherapy in most studies. Zhang have reported that higher tumor vessel density in the endoscopic biopsy specimen was correlated with better clinical effects of definite CRT in esophageal carcinoma (Zhang et al., 2006). Concerning antiangiogenic therapies, the reported data are similar, with higher pretreatment values associated with a higher response rate to antiangiogenic drugs (Han et al., 2010; Fournier et al., 2010). A possible explanation of this feature would be that well-perfused tumors allow delivery of chemotherapy and may have better oxygenation and thus potentially have greater radio-sensitivity. Thus, these data support our present observation that perfusion parameters will be useful predictive factors in patients who have undergone CRT.

To evaluate the predictive value of the perfusion parameters for the response, we used BF as the predictor of response to radiation and chemotherapy in the development of the prediction model. Our study demonstrated that $56 \%$ specificity and $94.4 \%$ sensitivity for tumor response in those patients with baseline BF more than $36.10 \mathrm{ml} / 100 \mathrm{~g} /$ min. Makari also found the tumor BF by perfusion CT could partly predict the effect of chemotherapy and CRT and survival for advanced esophageal cancer (Makari et al., 2007). Zima found that that chemotherapy is more effective in advanced squamous cell carcinoma of the upper aerodigestive tract with high BV than in those with low BV (Zima et al., 2007). Our study showed the possibility that perfusion $\mathrm{CT}$ could predict the response to CRT in esophageal squamous cell carcinoma. In the near future, we may be able to determine who should undergo surgery without CRT and who should undergo CRT before surgery in patients of advanced esophageal squamous cell carcinoma.

There is little information on the use of perfusion CT for predicting prognosis in esophagus cancer. We found that patients with high BF tumors survive significantly longer than those with low BF tumors. This showed that esophageal cancer with low BF has a high malignant potentiality regarding resistance to adjuvant therapy. But this conclusion derived from the local advanced patients. All those enrolled patients were T3-4 stage with involved esophageal wall thickness more than $10 \mathrm{~mm}$ in 
our study. For these patients, the persistent disease and local recurrence are the predominant failure patterns and negatively impacted survival more significantly. Since this is a preliminary observation, further study needs to be done.

As mentioned above, though there were marked differences with regard to tumor types, tumor size, treatment modalities, therapeutic agents, and perfusion CT technique and software, and even observer variability and the sample size of studies. Most of these results show that perfusion CT measurements are reproducible and elevated perfusion CT parameters are statistically correlated to a better response to radiotherapy and chemotherapy. We also found some discrepancies in the association between perfusion values and response to therapy in these studies. For example, the PS and MTT have not sufficiently predictive of the response in some studies. In further studies, a more standardized protocol should be used and large scale observations are needed to verify the value of each parameter in predicting of response.

Our study had some limitations. First, our patient numbers were small and inevitably reduced the power of our study. Another question, when response to therapy is analyzed, the $\mathrm{T}$ stage of the esophagus cancer is an important factor. Our study included patients with advanced $\mathrm{T}$ stage, which the involved esophagus was easily evaluated on CT scans. We are not sure these results are suitable for early stage patients, which has a higher response rate after definitive RT/CRT. Thirdly, we did not evaluate the changes of perfusion parameters during and after chemo-radiation therapy, which would demand a high patient compliance. Theoretically, a dynamic perfusion CT study could provide more comprehensive information about the treatment on tumor vascularity, which may reinforce its evidence for predicting response and survival. And a question must be mentioned, the analytical method used in the software of our study was based on the maximum slope method and the perfusion-measuring algorithms are not interchangeable between deconvolution-based and maximum slope-based algorithms (Djuric-Stefanovic et al., 2013).

In conclusion, our study demonstrated that perfusion $\mathrm{CT}$ is feasible for predicting tumor response and the prognosis in patients with advanced esophageal squamous cell carcinoma after radiotherapy or chemo-radiotherapy. This observation should be confirmed by large cohort studies in homogeneous patient groups.

\section{Acknowledgements}

This work was supported by a Grant for Project Research (ZR2012HL35) from Shandong Provincial Natural Science Foundation, China. We thank Dr. Peng $\mathrm{Xie}$ for assistance with the statistics analysis.

\section{References}

Bedenne L, Michel P, Bouché O, et al (2007). Chemoradiation followed by surgery compared with chemoradiation alone in squamous cancer of the esophagus: FFCD 9102. J Clin Oncol, 25, 1160-8.
Bellomi M, Petralia G, Sonzogni A, et al (2007). Perfusion CT for the monitoring of neoadjuvant chemotherapy and radiation therapy in rectal carcinoma:initial experience. Radiology, 244, 486-93.

Bisdas S, Rumboldt Z, Surlan-Popovic K, et al (2010). Perfusion $\mathrm{CT}$ in squamous cell carcinoma of the upper aerodigestive tract:long-term predictive value of baseline perfusion CT measurements. AJNR Am J Neuroradiol, 31, 576-81.

Chen TW, Yang ZG, Wang QL, et al (2011). Whole tumour quantitative measurement of first-pass perfusion of oesophageal squamous cell carcinoma using 64-row multidetector computed tomography:correlation with microvessel density. Eur J Radiol, 79, 218-23.

Djuric-Stefanovic A, Saranovic Dj, et al (2013). Comparison between the deconvolution and maximum slope 64-MDPerfusion CT analysis of the esophageal cancer: is conversion possible? Eur J Radiol, 82, 1716-23.

Fournier LS, Oudard S, Thiam R, et al (2010). Metastatic renal carcinoma: evaluation of anti-angiogenic therapy with dynamic contrast enhanced CT. Radiol, 256, 511-8.

Goh V, Padhani AR (2006). Imaging tumor angiogenesis: functional assessment using MDCT or MRI? Abdom Imaging, 31, 194-9.

Han KS, Jung DC, Choi HJ, et al (2010). Pretreatment assessment of tumor enhancement on contrastenhanced computed tomography as a potential predictor of treatment outcome in metastatic renal cell carcinoma patients receiving antiangiogenic therapy. Cancer, 116, 2332-42

Hayano K, Okazumi S, Shuto K, et al (2007). Perfusion CT can predict the response to chemoradiation therapy and survival in esophageal squamous cell carcinoma: initial clinical results. Oncol Rep, 18, 901-8.

Kelsen DP, Winter KA, Gunderson LL, et al (2007). Longterm results of RTOG trial 8911 (USA Intergroup 113): a randomassignment trial comparison of chemotherapy followed by surgery compared withsurgery alone for esophageal cancer. J Clin Oncol, 25, 3719-25.

Kersting S, Konopke R, Dittert D, et al (2009). Who profits from neoadjuvant radiochemotherapy for locally advanced esophageal carcinoma? J Gastroenterol Hepatol, 24, 886-95.

Laking GR, West C, Buckley DL, et al (2006). Imaging vascular physiology to monitor cancer treatment. Crit Rev Oncol Hematol, 58, 95-113

Li Y, Yang ZG, Chen TW, et al (2009). Quantitative assessment of first-pass perfusion of oesophageal squamous cell carcinoma using 64-section MDCT: initial observation. Clin Radiol, 64, 38-45.

Makari Y, Yasuda T, Doki Y, et al (2007). Correlation between tumor blood flow assessed by perfusion CT and effect of neoadjuvant therapy in advanced esophageal cancers. J Surg Oncol, 96, 220-9.

Miyata H, Yamasaki M, Takahashi T, et al (2014). Determinants of response to neoadjuvant chemotherapy for esophageal cancer using $18 \mathrm{~F}$-fluorodeoxiglucose positron emission tomography (18F-FDG-PET). Ann Surg Oncol, 21, 575-82.

Moulder JE and Rockwell S (1987). Tumor hypoxia: its impact on cancer therapy. Cancer Metastasis Rev, 5, 313-41.

Piessen G, Briez N, Triboulet JP, et al (2007). Patients with locally advanced esophageal carcinoma nonresponder to radiochemotherapy: who will benefit from surgery? Ann Surg Oncol, 14, 2036-44.

Stahl M, Wilke H, Stuschke M, et al (2005). Clinical response to induction chemotherapy predicts local control and longterm survival in multimodal treatment of patients with locally advanced esophageal cancer. J Cancer Res Clin Onco, 131, 67-72.

Tsai AG, Johnson PC and Intaglietta M (2003). Oxygen gradients 
Ming-Huan Li et al

in the microcirculation. Physiol Rev, 83, 933-63.

van Hagen P, Hulshof MC, van Lanschot JJ, et al (2012). Preoperative chemoradiotherapy for esophageal or junctional cancer. N Engl J Med, 366, 2074-84.

Wang DB, Zhang X, Han HL, et al (2012). Neoadjuvant chemoradiotherapy could improve survival outcomes for esophageal carcinoma: a meta-analysis. Dig Dis Sci, 57, 3226-33.

Yin LL, Yang ZG, Li Y, et al (2008). (Preliminary study of tumor perfusion assessment with multidetector CT in carcinoma of esophagus and cardia). Sichuan Da Хие Хие Ваo Yi Хие Ban, 39, 788-91.

Zhang SC, Hironaka S, Ohtsu A, et al (2006). Computerassisted analysis of biopsy specimen microvessels predicts the outcome of esophageal cancers treated with chemoradiotherapy. Clin Cancer Res, 12, 1735-42.

Zhu W, Xing L, Yue J, et al (2012). Prognostic significance of SUV on PET/CT in patients with localised oesophagogastric junction cancer receiving neoadjuvant chemotherapy/ chemoradiation:a systematic review and meta-analysis. $\mathrm{Br}$ J Radiol, 85, 694-701.

Zima A, Carlos R, Gandhi D, et al (2007). Can pretreatment $\mathrm{CT}$ perfusion predict response of advanced squamous cell carcinoma of the upper aerodigestive tract treated with induction chemotherapy? AJNR Am J Neuroradiol, 28, 328-34. 